\title{
Mechanistic Insights into Human Brain Impact Dynamics through Modal Analysis
}

\author{
Kaveh Laksari, ${ }^{1}$ Mehmet Kurt, ${ }^{2, *}$ Hessam Babaee, ${ }^{3}$ Svein Kleiven, ${ }^{4}$ and David Camarillo ${ }^{5}$ \\ ${ }^{1}$ Department of Bioemedical Engineering, University of Arizona, Tucson, Arizona 95719, USA \\ ${ }^{2}$ Department of Mechanical Engineering, Stevens Institute of Technology, Hoboken, New Jersey 07030, USA \\ ${ }^{3}$ Department of Mechanical Engineering and Materials Science, University of Pittsburgh, Pittsburgh, Pennsylvania 15261, USA \\ ${ }^{4}$ Division of Neuronic Engineering, KTH-Royal Institute of Technology, Huddinge 114 28, Sweden \\ ${ }^{5}$ Department of Bioengineering, Stanford University, Stanford, California 94305, USA
}

(Received 24 October 2016; revised manuscript received 26 October 2017; published 30 March 2018)

\begin{abstract}
Although concussion is one of the greatest health challenges today, our physical understanding of the cause of injury is limited. In this Letter, we simulated football head impacts in a finite element model and extracted the most dominant modal behavior of the brain's deformation. We showed that the brain's deformation is most sensitive in low frequency regimes close to $30 \mathrm{~Hz}$, and discovered that for most subconcussive head impacts, the dynamics of brain deformation is dominated by a single global mode. In this Letter, we show the existence of localized modes and multimodal behavior in the brain as a hyperviscoelastic medium. This dynamical phenomenon leads to strain concentration patterns, particularly in deep brain regions, which is consistent with reported concussion pathology.
\end{abstract}

DOI: 10.1103/PhysRevLett.120.138101

Traumatic brain injury (TBI) is a major cause of death and disability in the United States, contributing to about $30 \%$ of all injury-related deaths $[1,2]$. Every year, millions of Americans are diagnosed with TBI [3,4], 80\% of which are categorized as mild [2]. Undiagnosed cases, due to either lack of clinical expertise or underreporting, might be twice as high [5-8]. Given that mild TBI (MTBI), or concussion, has become a serious health concern in society, the burden of understanding and preventing it has become ever more indisputable for clinicians and physicists alike.

Efforts to model the brain's physics date back to the 1940s when Holbourn proposed the head as a mechanical system and explored the relation between the input to this system (in the form of head motion) to the output (in the form of relative brain displacement) $[9,10]$. Kornhauser proposed isodisplacement curves in a second-order springmass system representing relative brain displacement as a measure for classifying injury [11]. Others have also showed that, in different loading regimes, injury could be more sensitive to peak acceleration or maximum change in velocity or a combination of both $[12,13]$. Since then, many scientists have investigated the brain's response in severe scenarios of TBI with skull flexure [14,15], and more recently in mild scenarios with mostly inertial loading on the brain [16-18]. In particular, for helmeted sports, much of previous research has focused on brain deformation while assuming a rigid skull. In time, with the advances in imaging techniques, axonal injury, which requires excessive regional stretching of axons [19,20], has become one of the leading hypotheses behind the mechanism of concussions. Confirming this hypothesis, strain in the brain and specifically strain in the periventricular region of the brain - with the highest density of axon fibers-have been shown to correlate best with acute concussion and longterm neurological deficits [21-24]. However, dynamical behavior of the brain during rapid head motions with various amplitudes, durations, and directions, as well as the reason for higher susceptibility of these deep regions of the brain to strain are still largely unknown [22,25].

As a complex dynamical system with an intricate geometry, nonuniformly compliant boundary conditions and significantly inhomogeneous material properties, understanding the mechanical characteristics of the brain requires a multifaceted approach that takes into account both the spatial and temporal aspects of this system. A force impulse on the head creates nonlinear traveling shear waves inside the brain, which propagate at different speeds and attenuate at different rates, and can create localized strain concentrations at different regions of a linear [26] and nonlinear [27] viscoelastic medium. Gurdjian et al. observed that shear strains were developed within the brain, which were concentrated in the vicinity of the brain stem [28]. A similar behavior was observed in the corpus callosum region, which interfaces with stiff ventricular or membranous structures. Relatively rigid structures inside the head, e.g., falx cerebri and tentorium cerebelli, are also highly influential in reflecting or redirecting the shear waves $[29,30]$.

In addition to the spatial distribution of these shear waves, our understanding of their temporal dynamics is rather limited. This is an important gap in knowledge especially since impact biomechanics of the brain is by-and-large a transient phenomenon, spanning a few to a hundred milliseconds [22,31]. Previous studies have shown a strong dependence of brain motion and deformation on the 
frequency of the input loading [32-36]. Margulies et al. showed that the maximum strain induced in a brain surrogate material had a strong dependence on the frequency of the applied head motion with peak values occurring near $25 \mathrm{~Hz}$ [37,38]. Most recently, using a lumped-parameter model validated with tagged-MRI measurements and cadaveric impact experiments, we modeled the governing dynamics of the brain in the sagittal plane and discovered an amplified global behavior for relative brain motion at around $20 \mathrm{~Hz}$ [35].

In this Letter, we study the spatiotemporal characteristics of brain deformation during athletic events. By leveraging mode decomposition techniques, we demonstrate localized dynamical phenomena during brain tissue deformation and report on its implications on injury assessment and preventive equipment design.

Modal analysis is widely used in structural and fluid mechanics, as well as biomedical signal analysis to extract modal behavior of a data sequence in the absence of an underlying model [39-41]. Here we used dynamic mode decomposition (DMD), which is a multivariate method developed to reduce the dimensionality of a given timeseries data set [42]. DMD gives a compact representation of the original data set by computing a set of independent modes, each oscillating at a fixed frequency. It can give the system's characteristics such as energy and decay rate.

In order to understand how DMD works, we consider a temporal sequence of the brain's nodal displacement fields, $\mathcal{U}(x, y, z, t)$, where $x, y, z$ are the spatial location of each node considered at time $t$. Assume that we have $N$ equally spaced snapshots of $M$ nodes, which in this case translates to $3 \times M$ degrees of freedom in each snapshot. Using the DMD definition, we decompose the displacement fields into the following form:

$$
\mathcal{U}(x, y, z, t)=\sum_{n=1}^{N} a_{n} \exp \left(\lambda_{n} t\right) \phi_{n}(x, y, z)
$$

such that $\mathcal{U}_{1}^{N}=\left\{\mathbf{u}_{1}, \mathbf{u}_{2}, \ldots, \mathbf{u}_{N}\right\}$. Here, $a_{n}$ is the modal coefficient, $\lambda_{n}$ is the complex modulus, and $\phi_{n}$ is the spatial distribution of each mode. The main assumption in DMD is that each snapshot in time is assumed as a linear combination of the previous snapshots such that $\mathbf{u}_{j+1}=\mathbf{A} \mathbf{u}_{j}$, which in turn leads to $\mathcal{U}_{1}^{N}=\left\{u_{1}, \mathbf{A} u_{1}, \ldots, \mathbf{A}^{N-1} u_{1}\right\}$. The goal here is to extract the dynamic characteristics, i.e., eigenvalues and eigenvectors of the dynamical process described by $\mathbf{A}$, which is a large square matrix of size $3 M \times N$. As the number of grid nodes increases, so does the size of this matrix. In order to avoid forming this large matrix $\mathbf{A}$, a companion matrix $\tilde{\mathbf{S}}=H^{T} \mathbf{A} H$ is formed instead through singular value decomposition (SVD), where $H$ is the left singular vectors of $\mathcal{U}$. By using the eigenvectors of $\tilde{\mathbf{S}}$, the dynamic modes can be represented as

$$
\Psi=\mathcal{U}_{1}^{N-1} \mathbf{T},
$$

where $\mathbf{T}_{j}$ are the left eigenvectors of $\tilde{\mathbf{S}}$. In the following sections, we will utilize frequency $\left[\omega_{j}=\operatorname{Re}\left(\lambda_{n}\right)\right]$, decay rate $\left[\zeta_{j}=\operatorname{Im}\left(\lambda_{n}\right)\right]$, and amplitude $\left(\left|\Psi_{j}\right|\right)$ of these dynamic modes. $\lambda_{n}$ 's are calculated by using the eigenvalues of $\tilde{\mathbf{S}}$. For a more complete description of the DMD methodology, please refer to Refs. [42,43].

Other dimension reduction techniques such as proper orthogonal decomposition (POD) have been used in structural mechanics, where spatial structures of the modes are extracted based on the system's statistically steady-state response [44]. A crucial disadvantage of these energyranking methods is that, unlike DMD, they might lose valuable phase information since they utilize energy ranking schemes and are not able to give temporal and spatial separation [42]. We performed a quantitative comparison between DMD and POD and showed that for a highly transient event, such as a head impact during football games, DMD is better equipped to capture the presence of intrinsic nonlinearities in contrast to POD, which predicts invariant spatial substructures for different loading conditions (see Supplemental Material [45], Fig. S4).

To investigate the brain's response in real-world head impact conditions, we used our lab's previously published head kinematics from athletic events [22]. We have collected 537 head impact kinematics by instrumenting 31 athletes, two of whom sustained concussions: one suffered loss of consciousness (LOC), and the other selfreported subtle post-concussive symptoms. The recorded rotational and linear acceleration magnitudes and peak frequencies (representing impulse durations) span a large range with no apparent clustering patterns (see Supplemental Material [45], Figs. S1B,C). To capture the dynamic characteristics of the complex nonlinear brain-skull system, we randomly selected and simulated a subset of 187 noninjury collisions and the two injury collisions using an FE model developed at the KTH Royal Institute of Technology in Stockholm, Sweden (see Supplemental Material [45], videos S1 and S2 for the LOC case).

Having simulated the local brain tissue deformations with the above skull kinematics, we applied DMD to extract the dominant spatiotemporal characteristics of the brain's nodal displacements, which we can represent as a combination of modes that oscillate and decay at the corresponding modal frequencies and decay rates. As an example, we show the brain's modal behavior for the LOC case in Fig. 1. The first mode oscillates at $28 \mathrm{~Hz}$ and decays with a rate of $18.4 \mathrm{~s}^{-1}$, whereas the third mode oscillates at $42 \mathrm{~Hz}$ and decays with a rate of $29.4 \mathrm{~s}^{-1}$. Note that the displacements in the third mode appear and disappear approximately twice in the one cycle of the first mode shown here. The spatial distribution of the first and third modes are apparent in the snapshots, where the displacements initiate in the central region of the brain and propagate outwards in the form of a torus [Figs. 1(a) 


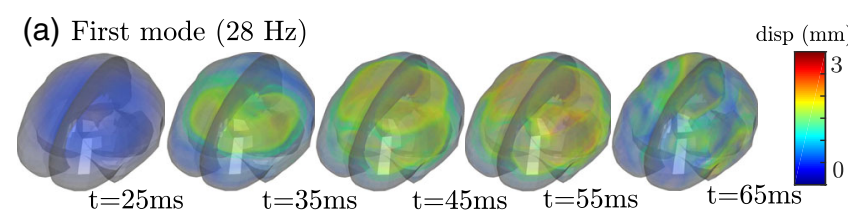

(b) Third mode $(42 \mathrm{~Hz})$

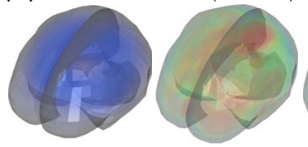

(c) Nodal displacements

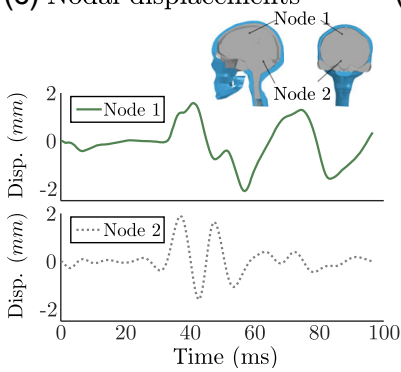

(d) Power spectral density

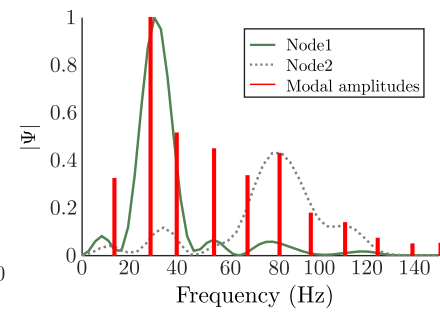

FIG. 1. Brain deformation due to a football head impact that led to loss of consciousness: (a) Propagation of the first displacement mode oscillating at $28 \mathrm{~Hz}$. (b) Propagation of the third displacement mode oscillating at $42 \mathrm{~Hz}$ showing out of phase oscillation with mode 1 (Supplemental Material [45], videos S3-S5). (c) Anterior-posterior displacement traces of two brain nodes; one at the interface between white and gray matter (node 1) and one from the cerebellum region (node 2). (d) Fourier modes of the displacement traces of the brain nodes superimposed on the energy amplitude of the DMD modes, showing close agreement.

and 1(b), Supplemental Material [45] Figs. S5 and videos S3-S5].

The correspondence of the Fourier modes with the DMD modes is also quite interesting as explained in Ref. [48]. Fourier analysis of two arbitrarily chosen nodes, one at the interface of white and gray matter (node 1), with low frequency dynamics, and one in the cerebellum (node 2), with higher frequency multimodal dynamics, shows the ability of DMD analysis to capture the Fourier modes of each node [Figs. 1(c) and 1(d)]. It should be noted that DMD has important advantages over Fourier analysis due to its applicability to nonlinear and transient systems. In the limit case of a linear system with periodic loading, DMD can be simplified to Fourier analysis [49]. Since our loading input is an impulse loading and our system is both nonlinear and highly rate dependent, DMD seems advantageous for modal decomposition.

We observed that in spite of the brain's geometrical complexity and material nonlinearity, in the span of the loading inputs studied here, several common characteristics emerge. The main observation from the DMD analysis was that the brain's displacement modes are amplified in a frequency regime around $30 \mathrm{~Hz}$ [Fig. 2(a)]. Here the amplified modal amplitudes indicate that the brain is more susceptible to vibrations in the $20-40 \mathrm{~Hz}$ range. In (a) Modal amplitudes

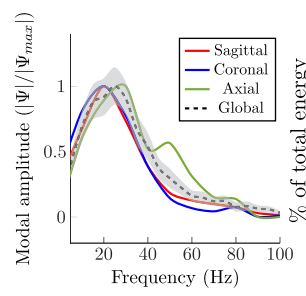

(b) Modal energies

(c) Peak principal strains
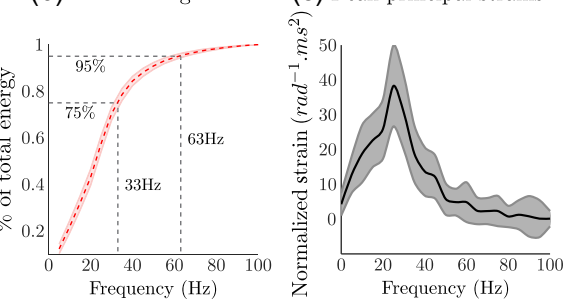

FIG. 2. Overall temporal characteristics of brain deformation: (a) Average modal amplitudes of all displacement modes of all the simulated case, showing amplification at $28 \mathrm{~Hz}$. Modes in the axial direction show a greater bandwidth than those in the sagittal and coronal directions. (b) Average accumulated modal energy for all simulated cases which correlates with the explained variance using each mode, showing exponential convergence to $100 \%$ variance. (c) Strain values derived from each mode normalized by the peak rotational acceleration of the corresponding head kinematics.

Fig. 2(a), the dashed line represents the mean values for all the simulations and the shaded region represents the standard error of the mean for the global brain modes. To compare the effect of the direction of head rotation on the frequency content of these modes, we categorized each football case with respect to their corresponding "dominant" rotation direction, defined as the direction with highest rotational acceleration. We observed that the frequency content of the sagittal and coronal modes were fairly similar, whereas axial rotation excited a larger frequency regime (up to $60 \mathrm{~Hz}$ ).

We further discovered that $75 \%$ of the total brain displacement energy can be captured by combining temporal modes with frequencies up to $33 \mathrm{~Hz}$, which indicates the shear waves at higher frequencies are less significant for the brain dynamics [Fig. 2(b)]. This could be an important consideration for developing reduced-order models that are capable of simulating the brain's response and achieving close to real-time analysis. The effect of this low frequency behavior is directly reflected in the tissue-level strains as well. To separate the effect of input amplitude from modal behavior, we normalized the principal strains with respect to peak rotational acceleration, as there is an almost linear relationship between the two (see Supplemental Material [45], Fig. S2). As we show in Fig. 2(c), the mean and standard error of peak principal strains normalized with respect to peak rotational acceleration exhibit amplifying behavior in the $20-40 \mathrm{~Hz}$ frequency regime, following a similar trend to the modal amplitudes. These results confirmed our previous assessment concerning the importance of the low frequency brain deformation [35].

So far, we have only examined the dominant mode, i.e., the mode with the highest amplitude, in each simulation; however, brain dynamics is influenced by the interaction of multiple modes that might have substantial contribution to tissue deformation. To quantify the extent of this 
contribution, we investigated how the peak principal strains calculated from the FE model compare against the predictions of the second-order mass-spring system proposed by Kornhauser [50]. The spring-mass system was used to approximate the contribution of the dominant mode to the relative brain displacement. We used the largest directional component of rotational acceleration as base excitation. We used previously published values for the moment of inertia [51], and based on the results shown in Fig. 2(a), we assumed a $28 \mathrm{~Hz}$ natural frequency.

The rationale here was that if the global mode is the main contributing factor to brain strain, we would expect the relation between the maximum relative displacement from the lumped model to correlate almost linearly with the peak principal strains from the FE model. The results are shown in Fig. 3(a), where the dashed line represents the best linear fit $\left(R^{2}=0.72\right)$ between peak principal strain and maximum displacement from the lumped model. As expected, for low brain strains $(<15 \%)$, this relationship maintains its linearity. As the brain strains become larger, the linear correlation decreases and more outliers start to emerge, which we attribute to stronger multimodal behavior in brain regions. Because of the limited number of high strain cases, we superimposed strain and displacement predictions for 58 (25 injury cases) previously reported National Football League (NFL) head impact cases (represented by gray crosses) [52]. To formally investigate this effect, we used the parameter dynamic range, which we defined as the span of frequencies that exist within $50 \%$ of the dominant mode's amplitude. The dynamic range increases from zero

(a) Modal behavior

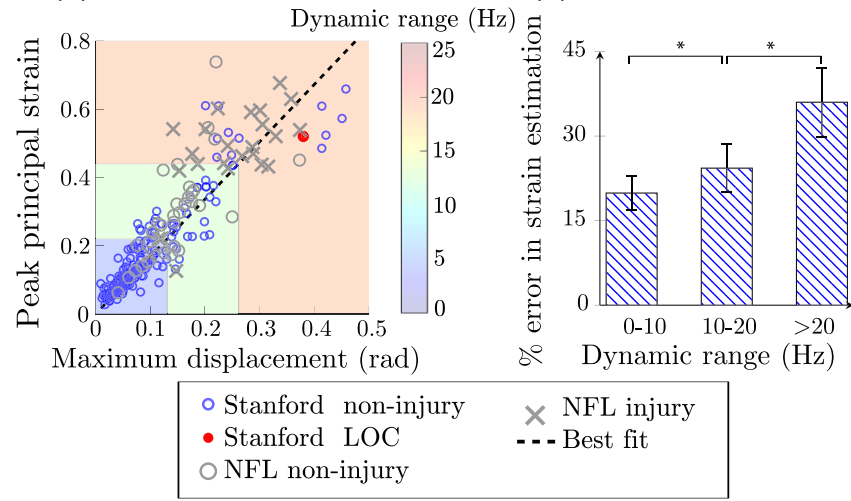

FIG. 3. Dynamic ranges of brain modes indicate multimodal behavior for higher input amplitudes: (a) Football data from Stanford mouthguard and NFL data from Ref. [52] are compared against the best linear fit calculated by using the global dominant mode [Fig. 4(a)]. Multimodal behavior is observed for higher strain values, where a single mode fails to predict the peak principal strain. The dynamic range of football cases reveals a transition towards a multimodal behavior at higher strain values. (b) The dynamic ranges of individual football simulations significantly increase as they are further away from the single mode prediction. to $25 \mathrm{~Hz}$ as we go towards higher strain levels. By grouping the strain values so that they fall within $10 \mathrm{~Hz}$ dynamic range bins, we see an increase in the average error for higher strains. As shown in Fig. 3(b), the correlation between the 1D model and the FE model's predictions decreases with increasing dynamic range, indicating richer dynamical behavior $(p<0.01)$.

The multimodal behavior of the brain presented here provides interesting insights into possible mechanistic causes of sports-related concussion. Almost all of the injury cases we examined fell within the highest dynamic range when compared with other football cases, signaling a strongly multimodal behavior. It is not surprising to see that most of this multimodal behavior falls into the higher strain regions since higher skull kinematics lead to higher tissue deformations, intensify the effects of nonlinear material behavior and geometric nonlinearities, and enrich the resultant dynamics in the frequency domain.

The richer temporal dynamics caused by the multimodal behavior has the potential to create more spatiotemporal differences within the brain structures, hence resulting in localized dynamical phenomena and exacerbating the regional relative displacements. The TBI community has long regarded the periventricular area of the brain to be a specifically vulnerable region during rapid head motions [53] and tissue deformation in this area has been proposed as a potential cause for concussion [22,54]. To explore this further, we examined the spatial variation of the dominant mode frequency within the brain for seven different brain structures spanning deep and cortical regions of the brain: corpus callosum (CC), gray matter (GM), brain stem (BS), midbrain $(\mathrm{MB})$, white matter (WM), thalamus $(\mathrm{TH})$, and cerebellum (CB) [Fig. 4(a)]. We compared the modal behavior for each region, between the LOC case and the average case, where we took the mean and standard deviation of all the 187 noninjury cases in both frequency and normalized strain measures [represented by horizontal and vertical error bars in Fig. 4(b)]. Superimposing the regional frequencies and normalized peak principal strains onto the global trends [repeated from Fig. 2(c)], we found that corpus callosum has a distinct modal frequency around $25 \mathrm{~Hz}$ and the corresponding contribution of this mode creates the highest strain levels in the brain, when compared with other regions. The local mode in CC for LOC has an even higher contribution to the tissue-level strains, resulting in 20\% higher normalized strains than the brain's average at $25 \mathrm{~Hz}$. Other brain regions have distinct modal frequencies as well, with the brain stem having the highest modal frequency around $40 \mathrm{~Hz}$. We did not observe clear differences in the variance of local decays rates between the two cases (see Supplemental Material [45], Fig. S6). The LOC case, which has a high dynamic range, leads to distinct local frequencies, specifically in the periventricular region. In contrast, the average case with a low dynamic range, expresses a smooth distribution of local frequencies. 
(a) Schematic of brain model

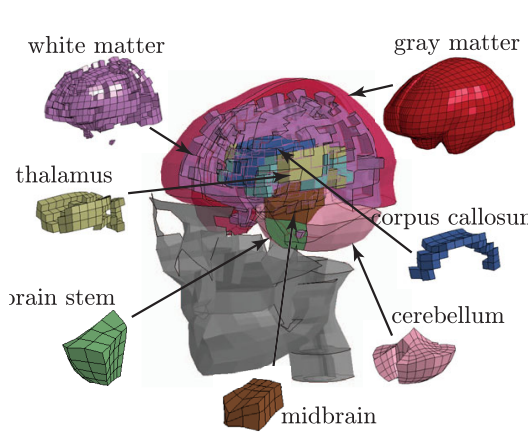

(b) Regional frequencies

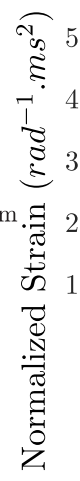

(c) Dominant frequency - LOC (d) Dominant frequency - average

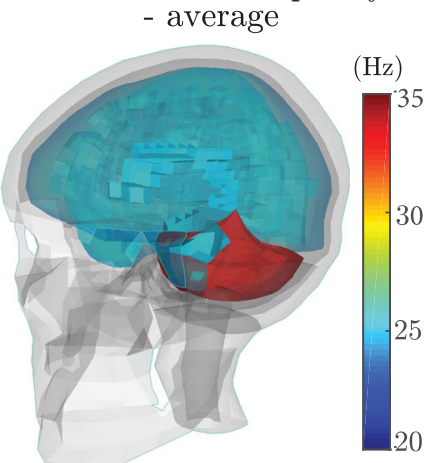

FIG. 4. Dominant frequencies of brain structures: (a) Schematic of the FE model of the head showing various parts of the brain. (b) Strain values derived from each mode normalized by the peak rotational acceleration of the corresponding head kinematics. Here, we superimpose regional dominant frequencies for each brain part for the LOC case (red circles) and the average and standard deviations (blue solid dots) for all cases. Brain part abbreviations: corpus callosum (CC), gray matter (GM), brain stem (BS), midbrain (MB), white matter (WM), thalamus (TH), corpus callosum, cerebellum (CB). (c) Structural distribution of the dominant frequency of brain regions for the LOC case, indicating out of phase oscillation due to significant discrepancy between regions especially in the periventricular region. (d) Structural distribution of dominant frequencies for an average head impact case, which indicates a lower dynamic range.

The increased localization in CC for the LOC case compared with the average case is noteworthy, especially since this region was identified as a good correlate for concussion classification in our previous study [22] [Figs. 4(b)-4(d)]. These results suggest a potential link between mode localization and tissue damage.

A complete investigation of the effect of multimodal behavior on regional brain dynamics requires a more extensive study; however, a closer examination of the LOC head impact case against the average modal behavior supports our injury hypothesis above: localized dynamics caused by the multimodal behavior of the brain tends to excite more dangerous dynamics in the deep brain structures.

From a mechanistic point of view, understanding the initiation of these localized modes requires taking into account the nonlinear material properties and geometry of the skull-brain system. Even in the absence of skull deformation, external forces on the head will translate to the brain tissue. Given the compliance of the skull-brain interface $[55,56]$ and the viscoelastic and nonlinear behavior of the brain tissue [57], this energy translation will lead to the initiation of shear and longitudinal waves inside the brain. Motion and deformation of the brain under external loading, which is governed by damped hyperbolic equations, will have nonlinear shear and longitudinal components $[27,58,59]$. The Zabolotskaya equation describes the propagation of finite-amplitude, two-dimensional, linearly polarized, shear waves in nonlinear solids [60]. Let $U(x, y, t)$ depict the 2D displacement field in a nonlinear media and $x$ be the direction of shear wave propagation. The shear wave propagation can then be represented as follows [61]:

$$
\left(U_{t}+\beta U^{2} U_{x}\right)_{x}+\frac{1}{2} U_{y y}=0,
$$

where $\beta$ is a parameter that characterizes the material properties of the solid. The cubic nonlinear term encountered in the above equation is known to generate localized nonlinear modes in structural systems with geometric nonlinearities [62,63], systems with nonlinear absorbers or energy sinks [64], and granular dimer chains [65,66]. Localization and motion confinement are observed when vibrational energy tends to be confined to one particular area of a structure or continuous media. They have first been observed for periodic linear structures presenting a structural irregularity [67]. A generic property of nonlinear mode localization is high localized amplitudes in a particular area of the media with a distinct oscillation frequency. In this study, through the use of dynamic mode decomposition, we have found evidence regarding the existence of such modes as well as strain localization in the brain, particularly in the deep white matter brain regions. To the best of our knowledge, there has been no experimental evidence regarding the existence of such modes in nonlinear viscoelastic media up to date. By combining experimental measurements with computational modeling, our study presents the first indirect empirical evidence regarding the existence of localized modes with varying eigenvalues in a nonlinear, viscoelastic, and inhomogeneous media.

In summary, we used the FE model to simulate 187 head impacts collected during football game and practice. This provided over 40000 snapshots of over 5000 nodal brain displacements. We utilized DMD analysis to extract modal behavior of the brain from these highly transient events. Our findings show that (i) the brain-skull system is dominated by low frequency dynamics and more than $75 \%$ of the brain's displacement can be captured by temporal modes with frequencies under $33 \mathrm{~Hz}$, (ii) strain in brain tissue is amplified in frequencies close to $30 \mathrm{~Hz}$, (iii) high dynamic range and multimodal behavior might be 
associated with higher risks of injury, and (iv) periventricular regions of the brain are prone to mode localization, which is the first such evidence for this type of phenomenon in a nonlinear hyperviscoelastic medium.

There are several limitations associated with the work presented here. The FE model used in this study had previously been partially validated with sparse cadaver experiments and not thoroughly validated against in vivo MRI data $[25,68]$. We believe that more accurate and region specific material properties and more detailed geometries, particularly the cortical gyri, will render the dynamic differences more pronounced. The constitutive material models used in the FE simulations incorporate a linear viscosity definition, which might explain why the local decay rates in LOC and average cases were similar (see Supplemental Material [45], Fig. S6). In addition, the loading space used in this study is limited to head impacts in football. Although similar results might be expected in higher loading regimes, such as car accidents, the loading bias in different anatomical regions might have a significant effect on the resulting brain dynamics. Also, we had relatively low number of axial rotation cases from our football data compared to coronal and sagittal, which might render the frequency regimes of the axial modes [Fig. 2(a)] less accurate. Finally, there are small errors in the mouthguard measurements, previously quantified in anthropomorphic test dummy and in vivo video-tracking experiments [69,70].

The spatiotemporal characteristics of the human brain we identified in this Letter are particularly interesting because strain in corpus callosum has previously been shown to be the best predictor for concussion cases in football $[17,22]$. Similar observations have been made in previous diffusion tensor imaging studies to detect brain abnormalities in patients with concussions. Fiber tracts in the deep brain structures such as corpus callosum, fornix, hippocampus, thalamus, and cingulum bundles have been shown to be the most common locations of abnormal fractional anisotropy and mean diffusivity [71]. There needs to be more clinical observations to corroborate with the findings of this study.

With the advancing imaging technology in acquiring more spatially and temporally resolved experimental data, and more detailed finite element models, it is conceivable that ever larger data sets will become available in the future. As such, our proposed methodology is particularly helpful in extracting most notable characteristics of brain dynamics. A natural next step would be to use this technique in developing reduced-order models of brain deformation that could be used to perform near real-time simulations of head impacts, providing crucial information to clinicians for informed return-to-play decisions.

This research was supported by Child Health Research Institute, Lucile Packard Foundation for Children's Health, Stanford CTSA (UL1 TR001085) and Thrasher Research Foundation, who funded this work through the Thrasher Early Career Award. This study was also partially supported by the National Institutes of Health (NIH) (Grant No. 3R21EB01761101S1) National Institute of Bio-medical Imaging and Bioengineering (NIBIB) 3R21EB01761101S1. We also thank Dr. Andrew Knutsen and Dr. Philip Bayly for sharing the MRI data.

K. Laksari and M. Kurt contributed equally to this work.

*mkurt@stevens.edu

[1] M. Faul, L. Xu, M. M. Wald, and V. Coronado, Atlanta, GA: Centers for Disease Control and Prevention, National Center for Injury Prevention and Control (2010), https://stacks.cdc .gov/view/cdc/5571.

[2] C. L. MacDonald, A. M. Johnson, E. C. Nelson, N. J. Werner, R. Fang, S. F. Flaherty, and D. L. Brody, J. Neurotrauma 31, 889 (2014).

[3] J. A. Langlois, W. Rutland-Brown, and M. M. Wald, J. Head Trauma Rehabilitation 21, 375 (2006).

[4] Centers for Disease Control and Prevention, MMWR Morb Mortal Wkly Rep. 60, 1337 (2011).

[5] M. McCrea, T. Hammeke, G. Olsen, P. Leo, and K. Guskiewicz, Clinical J. Sport Medicine 14, 13 (2004).

[6] J. G. Garrick, Clinical journal of sport medicine 15, 385 (2005).

[7] I. J.S. Williamson and D. Goodman, British Journal of Sports Medicine 40, 128 (2006).

[8] S. R. Laker, PM\&R 3, S354 (2011).

[9] A. Holbourn, Lancet 242, 438 (1943).

[10] A. Holbourn, J. Neurosurg. 1, 190 (1944).

[11] M. Kornhauser, J. Appl. Mech. 21, 371 (1954).

[12] A. Ommaya and A. E. Hirsch, J. Biomech. 4, 13 (1971).

[13] S. Margulies and L. Thibault, J. Biomech. 25, 917 (1992).

[14] J. Versace, SAE Technical Paper 710881, 1971, https://www .sae.org/publications/technical-papers/content/710881/.

[15] W. C. Moss, M. J. King, and E. G. Blackman, Phys. Rev. Lett. 103, 108702 (2009).

[16] L. Zhang, K. H. Yang, and A. I. King, J. Biomech. Eng. 126, 226 (2004).

[17] S. Kleiven, Int. J. Crashworthiness 11, 65 (2006).

[18] B. Coats, S. A. Eucker, S. Sullivan, and S. S. Margulies, Int. J. Dev. Neuroscience: The Official Journal of the International Society for Developmental Neuroscience 30, 191 (2012).

[19] T. M. Talavage, E. a. Nauman, E. L. Breedlove, U. Yoruk, A. E. Dye, K. E. Morigaki, H. Feuer, and L. J. Leverenz, J. Neurotrauma 31, 327 (2014).

[20] J. J. Bazarian, T. Zhu, J. Zhong, D. Janigro, E. Rozen, A. Roberts, H. Javien, K. Merchant-Borna, B. Abar, and E. G. Blackman, PLoS One 9, e94734 (2014).

[21] S. Kleiven, Stapp Car Crash J. 51, 81 (2007).

[22] F. Hernandez, L. C. Wu, M. C. Yip, K. Laksari, A. R. Hoffman, J. R. Lopez, G. A. Grant, S. Kleiven, and D. B. Camarillo, Ann. Biomed. Eng. 43, 1918 (2015).

[23] S. Sullivan, S. A. Eucker, D. Gabrieli, C. Bradfield, B. Coats, M. R. Maltese, J. Lee, C. Smith, and S. S. Margulies, Biomech. Model. Mechanobiol. 14, 877 (2015).

[24] C. Giordano and S. Kleiven, SAE Technical Paper 201422-0002, 2014, https://www.sae.org/publications/technicalpapers/content/2014-22-0002/. 
[25] A. K. Knutsen, E. Magrath, J. E. McEntee, F. Xing, J. L. Prince, P. V. Bayly, J. A. Butman, and D. L. Pham, J. Biomech. 47, 3475 (2014).

[26] P. G. Massouros, P. V. Bayly, and G. M. Genin, Int. J. Solids Struct. 51, 305 (2014).

[27] B. Giammarinaro, D. Espíndola, F. Coulouvrat, and G. Pinton, Phys. Rev. Applied 9, 014011 (2018).

[28] E. Gurdjian, V. Hodgson, L. Thomas, and L. Patrick, J. Neurosurg. 29, 70 (1968).

[29] A. A. Sabet, E. Christoforou, B. Zatlin, G. M. Genin, and P. V. Bayly, J. Biomech. 41, 307 (2008).

[30] E. H. Clayton, G. M. Genin, and P. V. Bayly, J. R. Soc. Interface 9, 2899 (2012).

[31] S. Rowson, G. Brolinson, M. Goforth, D. Dietter, and S. Duma, J. Biomech. Eng. 131, 061016 (2009).

[32] A. K. Ommaya and T. A. Gennarelli, Brain 97, 633 (1974).

[33] H. Zou, S. Kleiven, and J. P. Schmiedeler, Int. J. Crashworthiness 12, 341 (2007).

[34] N. Yoganandan, J. Li, J. Zhang, F. A. Pintar, and T. A. Gennarelli, J. Biomech. 41, 2253 (2008).

[35] K. Laksari, L. C. Wu, M. Kurt, C. Kuo, and D. C. Camarillo, J. R. Soc. Interface 12, 20150331 (2015).

[36] L. C. Wu, K. Laksari, C. Kuo, J. F. Luck, S. Kleiven, C. R. Bass, and D. B. Camarillo, J. Biomech. 49, 2918 (2016).

[37] S. S. Margulies and L. E. Thibault, J. Biomech. Eng. 111, 241 (1989).

[38] S. S. Margulies, L.E. Thibault, and T. A. Gennarelli, J. Biomech. 23, 823 (1990).

[39] M. Costa, A. L. Goldberger, and C. K. Peng, Phys. Rev. Lett. 95, 198102 (2005).

[40] M. Kurt, H. Chen, Y. S. Lee, D. M. McFarland, L. A. Bergman, and A. F. Vakakis, Archive of Applied Mechanics 82, 1461 (2012).

[41] M. Grilli, A. Vazquez-Quesada, and M. Ellero, Phys. Rev. Lett. 110, 174501 (2013).

[42] P. J. Schmid, J. Fluid Mech. 656, 5 (2010).

[43] M. Grilli, P. J. Schmid, S. Hickel, and N. A. Adams, J. Fluid Mech. 700, 16 (2012).

[44] G. Kerschen, J. C. Golinval, A. F. Vakakis, and L. A. Bergman, Nonlinear Dyn. 41, 147 (2005).

[45] See Supplemental Material at http://link.aps.org/ supplemental/10.1103/PhysRevLett.120.138101 for a description of the head kinematics measurements, motivation for decomposition methods on tagged MRI measurements, and the finite element model, which includes Refs. [46,47].

[46] D. Camarillo, P. Shull, and J. Mattson, Ann. Biomed. Eng. 41, 1939 (2013).

[47] W. Hardy, M. Mason, C. Foster, C. S. Shah, J. M. Kopacz, K. H. Yang, and A. I. King, Stapp Car Crash J. 51, 17 (2007).

[48] C. W. Rowley, I. Mezić, S. Bagheri, P. Schlatter, and D. S. Henningson, J. Fluid Mech. 641, 115 (2009).

[49] K. K. Chen, J. H. Tu, and C. W. Rowley, J. Nonlinear Sci. 22, 887 (2012).
[50] M. Kornhauser and A. Gold, in Impact Acceleration Stress (National Research Council, Washington, DC, 1961), pp. 333-344.

[51] N. Yoganandan, F. a. Pintar, J. Zhang, and J. L. Baisden, J. Biomech. 42, 1177 (2009).

[52] E. Pellman, D. Viano, A. Tucker, and I. Casson, Neurosurgery 53, 796 (2003).

[53] V. E. Johnson, W. Stewart, and D. H. Smith, Exp. Neurol. 246, 35 (2013).

[54] T. W. McAllister, L. a. Flashman, A. Maerlender, R. M. Greenwald, J. G. Beckwith, T. D. Tosteson, J. J. Crisco, P. G. Brolinson, S. M. Duma, A.-C. Duhaime, M. R. Grove, and J. H. Turco, Neurology 78, 1777 (2012).

[55] S. Kleiven and W. N. Hardy, Stapp Car Crash J. 46, 123 (2002).

[56] W. Zhao and S. Ji, Interface Focus 6, 20150091 (2015).

[57] K. Laksari, M. Shafieian, and K. Darvish, J. Biomech. 45, 642 (2012).

[58] K. Laksari, K. Sadeghipour, and K. Darvish, J. Mech. Behavior of Biomedical Materials 32C, 132 (2013).

[59] K. Laksari, S. Assari, B. Seibold, K. Sadeghipour, and K. Darvish, Biomech. Model. Mechanobiol. 14, 459 (2015).

[60] D. L. Kraitchman, S. Sampath, E. Castillo, J. A. Derbyshire, R. C. Boston, D. A. Bluemke, B. L. Gerber, J. L. Prince, and N. F. Osman, Circulation 107, 2025 (2003).

[61] M. S. Cramer and M. F. Andrews, J. Acoust. Soc. Am. 114, 1821 (2003).

[62] M. Kurt, M. Eriten, D. M. McFarland, L. A. Bergman, and A. F. Vakakis, J. Sound Vib. 357, 331 (2015).

[63] E. Pesheck, N. Boivin, C. Pierre, and S. W. Shaw, Nonlinear Dyn. 25, 183 (2001).

[64] A. F. Vakakis, O. V. Gendelman, L. A. Bergman, D. M. McFarland, G. Kerschen, and Y. S. Lee, Nonlinear Targeted Energy Transfer in Mechanical and Structural Systems (Springer Science \& Business Media, New York, 2008), Vol. 156, http://www.springer.com/us/book/9781402091254.

[65] K. R. Jayaprakash, Y. Starosvetsky, and A. F. Vakakis, Phys. Rev. E 83, 036606 (2011).

[66] K. R. Jayaprakash, Y. Starosvetsky, A. F. Vakakis, and O. V. Gendelman, J. Nonlinear Sci. 23, 363 (2013).

[67] G. Kerschen, M. Peeters, J. C. Golinval, and A. F. Vakakis, Mechanical Systems and Signal Processing 23, 170 (2009).

[68] Y. Feng, T. M. Abney, R. J. Okamoto, R. B. Pless, G. M. Genin, and P. V. Bayly, J. R. Soc. Interface 7, 1677 (2010).

[69] L. C. Wu, V. Nangia, K. Bui, B. Hammoor, M. Kurt, F. Hernandez, C. Kuo, and D. B. Camarillo, Ann. Biomed. Eng. 44, 1234 (2016).

[70] C. Kuo, L. C. Wu, B. T. Hammoor, J. F. Luck, H. C. Cutcliffe, R. C. Lynall, J. R. Kait, K. R. Campbell, J. P. Mihalik, C. R. Bass, and D. B. Camarillo, J. Biomech. 49, 1845 (2016).

[71] M. Hulkower, D. Poliak, S. Rosenbaum, M. Zimmerman, and M. L. Lipton, Am. J. Neuroradiol. 34, 2064 (2013). 